University of Nebraska - Lincoln

DigitalCommons@University of Nebraska - Lincoln

Faculty Publications from the Department of Electrical \& Computer Engineering, Department Electrical and Computer Engineering

4-15-1991

\title{
Optical and magneto-optical characterization of TbFeCo thin films in trilayer structures
}

\author{
William A. McGahan \\ University of Nebraska-Lincoln \\ Ping $\mathrm{He}$ \\ University of Nebraska-Lincoln \\ Liang-Yao Chen \\ University of Nebraska-Lincoln \\ Sal Bonafede \\ University of Nebraska-Lincoln \\ John A. Woollam \\ University of Nebraska-Lincoln, jwoollam1@unl.edu \\ See next page for additional authors
}

Follow this and additional works at: https://digitalcommons.unl.edu/electricalengineeringfacpub

Part of the Electrical and Computer Engineering Commons

McGahan, William A.; He, Ping; Chen, Liang-Yao; Bonafede, Sal; Woollam, John A.; Sequeda, F.; McDaniel, T.; and Do, H., "Optical and magneto-optical characterization of TbFeCo thin films in trilayer structures" (1991). Faculty Publications from the Department of Electrical and Computer Engineering. 56. https://digitalcommons.unl.edu/electricalengineeringfacpub/56

This Article is brought to you for free and open access by the Electrical \& Computer Engineering, Department of at DigitalCommons@University of Nebraska - Lincoln. It has been accepted for inclusion in Faculty Publications from the Department of Electrical and Computer Engineering by an authorized administrator of DigitalCommons@University of Nebraska - Lincoln. 


\section{Authors}

William A. McGahan, Ping He, Liang-Yao Chen, Sal Bonafede, John A. Woollam, F. Sequeda, T. McDaniel, and $\mathrm{H}$. Do 


\title{
Optical and magneto-optical characterization of TbFeCo thin films in trilayer structures
}

\author{
William A. McGahan, Ping He, Liang-Yao Chen, Sal Bonafede, and John A. Woollam \\ Center for Microelectronic and Optical Materials Research and Departments of Electrical Engineering and \\ Physics, University of Nebraska-Lincoln, Lincoln, Nebraska 68588-0511
}

F. Sequeda, ${ }^{\text {a) }}$ T. McDaniel, and H. Do

IBM Storage Systems Products Division, San Jose, California 95193

\begin{abstract}
A series of TbFeCo films ranging in thickness from 100 to $800 \AA$ have been deposited in trilayer structures on silicon wafer substrates, with $\mathrm{Si}_{3} \mathrm{~N}_{4}$ being employed as the dielectric material. These films have been characterized both optically and magneto-optically by variable angle of incidence spectroscopic ellipsometry, normal angle of incidence reflectometry, and normal angle of incidence Kerr spectroscopy. From these measurements, the optical constants $n$ and $k$ have been determined for the TbFeCo films, as well as the magneto-optical constants $Q 1$ and $Q 2$. Results are presented that demonstrate the lack of dependence of these constants on the thickness of the TbFeCo film, and which can be used for calculating the expected optical and magneto-optical response of any multilayer structure containing similar $\mathrm{TbFeCo}$ films.
\end{abstract}

\section{INTRODUCTION}

Of all materials currently of interest for magnetooptical storage systems, the family of $\mathrm{TbFeCo}$ amorphous alloys is perhaps the most promising. ${ }^{1,2}$ This work was motivated by the desire to obtain accurate values of both the optical and magneto-optical constants of thin films of $\mathrm{TbFeCo}$ deposited in trilayer structures containing dielectric films of $\mathrm{Si}_{3} \mathrm{~N}_{4}$. The other primary purpose of this study was to determine the dependence (if any) of the optical and magneto-optical constants of the $\mathrm{TbFeCo}$ layers on the film thickness. Previous results from the Dy/Co compositionally modulated alloys suggested the possibility of variation in optical and magneto-optical properties from thickfilm values when thicknesses of less than $\sim 300 \AA$ are used. $^{3}$ As film thicknesses of the order of 100-800 $\AA$ are often desired for optimal recording structures, TbFeCo films spanning this range were studied in order to evaluate the dependence of the optical and magneto-optical response of the films on the thickness. Finally, this work was motivated by the desire to demonstrate the accurate characterization of magneto-optic thin films deposited in relatively complicated multilayer systems using readily available reflection mode experimental techniques, whereas this approach has been applied previously only to simpler systems. ${ }^{4,5}$

\section{SAMPLE PREPARATION}

Amorphous $\mathrm{TbFeCo}$ films were prepared by nonbiased dc magnetron sputtering techniques using commercially available alloy targets in an in-line sputtering system. The composition of the films, as determined by $x$-ray flourescence, was $20.33 \% \mathrm{~Tb}, 71.7 \% \mathrm{Fe}, 7.96 \%$ Co. Trilayer structures were fabricated on silicon wafer substrates $\left(\mathrm{Si} / \mathrm{Si}_{3} \mathrm{~N}_{4} / \mathrm{TbFeCo} / \mathrm{Si}_{3} \mathrm{~N}_{4}\right.$ ). The nominal thickness of the

\footnotetext{
a) On assignment from IBM Almaden Research Center.
}

bottom $\mathrm{Si}_{3} \mathrm{~N}_{4}$ layer (between the silicon substrate and the TbFeCo film) was $400 \AA$ while the top $\mathrm{Si}_{3} \mathrm{~N}_{4}$ layer was nominally $260 \AA$ thick for all samples. The nominal TbFeCo thickness was varied by $100-\AA \AA$ increments from 100 to $800 \AA$ A. All samples were found through Kerr loop measurements to have square hysteresis loops in the perpendicular orientation, with room-temperature coercivities ranging from $3.7 \mathrm{kOe}$ for the thinnest $\mathrm{TbFeCo}$ layer to 7.5 kOe for the thickest. Additionally, three films of $\mathrm{Si}_{3} \mathrm{~N}_{4}$, ranging from 200 to $800 \AA$ thick, were prepared on silicon substrates for determination of the $\mathrm{Si}_{3} \mathrm{~N}_{4}$ optical constants.

\section{EXPERIMENTAL PROCEDURE}

The samples were characterized optically by variable angle of incidence spectroscopic ellipsometry (VASE), which was performed over the spectral range 3000-8000 $\AA$ and at three angles of incidence: $60^{\circ}, 65^{\circ}$, and $70^{\circ} .{ }^{6.7}$ In addition, the intensity reflectance of each sample was measured at normal incidence over the spectral range 3800 $7500 \AA$. The samples were magneto-optically characterized

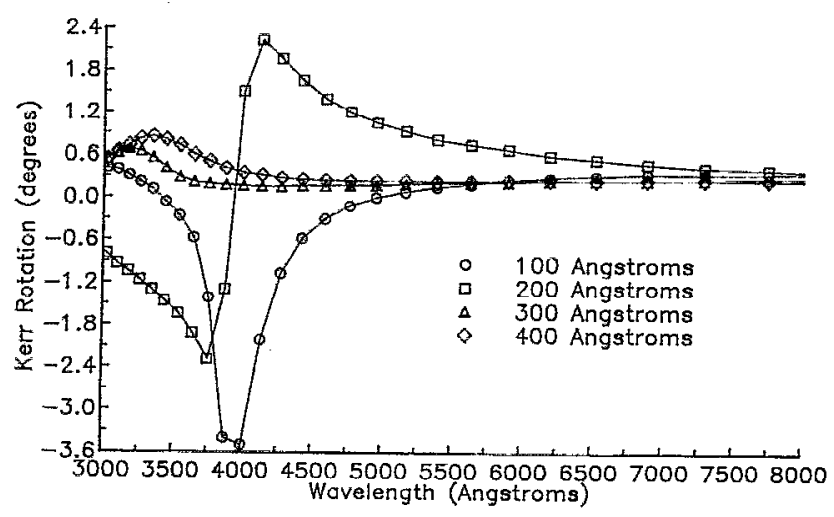

FIG. 1. Polar Kerr rotation measured at normal incidence for four TbFeCo films. 


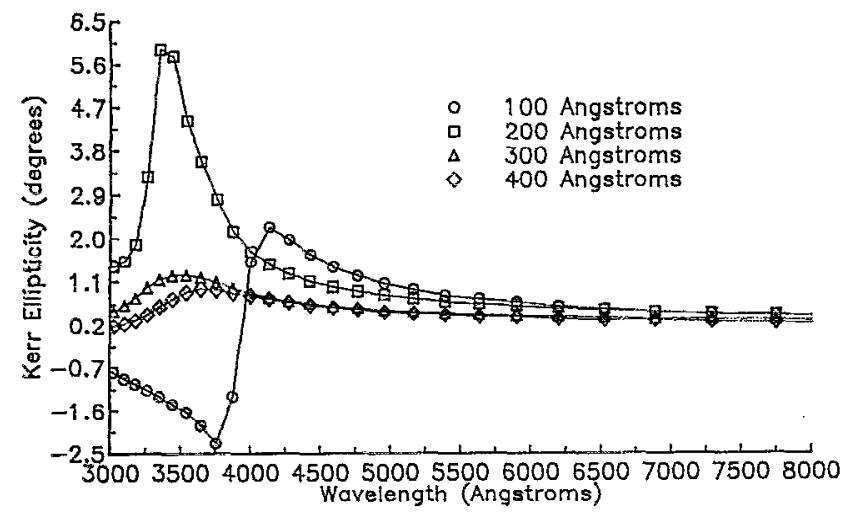

FIG. 2. Polar Kerr ellipticity measured at normal incidence for four TbFeCo films.

by measurement of the polar Kerr rotation and ellipticity at normal incidence over the spectral range $3000-8000 \AA .8$ Experimental Kerr data are shown in Figs. $1-3$, as a function of TbFeCo layer thickness. Spectra are shown for the first four layer thicknesses only, as both the Kerr parameters (as well as the reflectance) were found to be insensitive to increasing $\mathrm{TbFeCo}$ layer thickness for thicknesses in excess of $\sim 400 \AA$.

In order to improve the accuracy of the analysis the silicon substrate and $\mathrm{Si}_{3} \mathrm{~N}_{4}$ optical constants were measured by independent experiments. First, a silicon substrate was characterized ellipsometrically, and the thickness of the native oxide on a typical wafer was determined to be 23 $\AA$. Then, three samples of thin films $(200-800 \AA)$ of $\mathrm{Si}_{3} \mathrm{~N}_{4}$ were characterized ellipsometrically in order to determine the index of refraction of the $\mathrm{Si}_{3} \mathrm{~N}_{4}$ over the spectral range $3000-8000 \AA$. Given this information, nonlinear regression was used to determine the optical and magnetooptical constants of the $\mathrm{TbFeCo}$ layer in the trilayer structures, as well as the thickness of the top dielectric layer (and the storage and bottom dielectric layers when possible). This analysis was performed by using the LevenbergMarquardt algorithm to find the values of the unknown

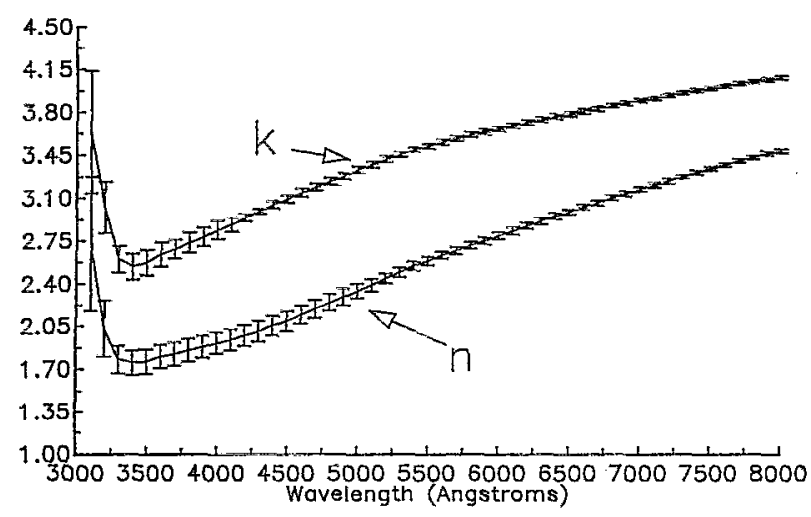

FIG. 3. Index of refraction $n$ and extinction coefficient for the TbFeCo films, found from simultaneous analysis of ellipsometric, reflectance, and Kerr data. Error bars represent spread of values for the entire range of layer thicknesses, not experimental error or statistical uncertainty.

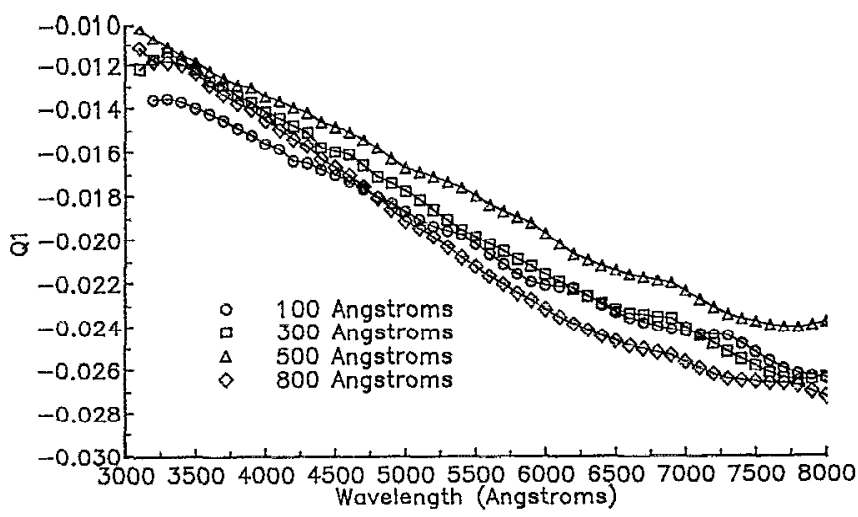

FIG. 4. Real part of the Voigt parameter $Q 1$, found from simultaneous analysis of ellipsometric, reffectance, and Kerr data. Data are shown for four different $\mathrm{TbFeCo}$ layer thicknesses.

parameters that minimized the mean-square error between the experimental data points and their calculated values. For this calculation, the $4 \times 4$ characteristic matrix formulation $^{9}$ was used to determine both the optical and magneto-optical response of the four-layer system ( $\mathrm{Si} / \mathrm{SiO}_{2} / \mathrm{Si}_{3} \mathrm{~N}_{4} / \mathrm{TbFeCo} / \mathrm{Si}_{3} \mathrm{~N}_{4}$ ). In this model, we assume that the complex index of refraction is given by $\tilde{n} \equiv n+i|k|$. The Voigt parameter is defined as the constant of proportionality between the diagonal and off-diagonal elements of the permitlivity tensor for a magneto-optic material, so that the permittivity tensor has the form

$$
[\widetilde{\epsilon}]=\left[\begin{array}{ccc}
\widetilde{\epsilon} & -i \widetilde{Q} \widetilde{\epsilon} & 0 \\
i \widetilde{Q} \widetilde{\epsilon} & \widetilde{\epsilon} & 0 \\
0 & 0 & \widetilde{\epsilon}
\end{array}\right],
$$

where $\widetilde{\epsilon}$, the isotropic dielectric constant, is equal to the square of the (complex) index of refraction.

The end point of this model calculation is the pseudoJones matrix for the multilayer system, whose elements are $\widetilde{R}_{p}$ and $\widetilde{R}_{s}$, the optical pseudo-Fresnel coefficients that describe the reflection of $p$ - and $s$-polarized light from the structure, respectively, and $\widetilde{K}$, the off-diagonal pseudo-

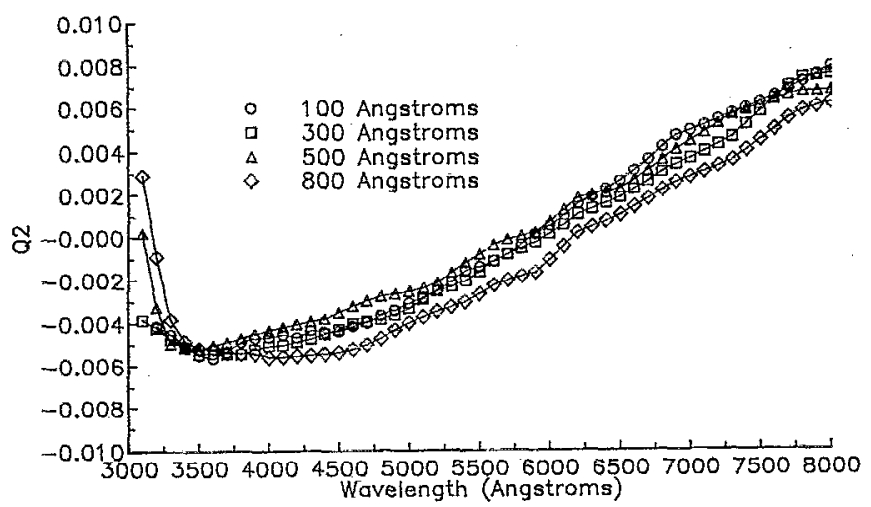

FIG. 5. Imaginary part of the Voigt parameter $Q 2$, found from simultaneous analysis of ellipsometric, reflectance, and Kerr data. Data are shown for four different $\mathrm{TbFeCo}$ layer thicknesses. 
Fresnel coefficient that determines the mixing of the $p$ - and $s$-polarized components of the incident beam upon reflection, and hence the magneto-optic activity of the sample. These coefficients can be calculated provided the optical and magneto-optical constants of each layer, as well as its thickness, are given, and the angle of incidence is known. Once the pseudo-Jones matrix has been found, the various experimental quantities can be calculated:

Reflectance:

$R=\left|\widetilde{R}_{p}\right|^{2}=\left|\widetilde{R}_{s}\right|^{2}$,

Kerr effect:

$$
o_{k}+i \eta_{k}=\widetilde{K} / \widetilde{R}_{s}=-\widetilde{K} / \widetilde{R}_{p}
$$

ellipsometric parameters:

$$
\begin{aligned}
& \tan \psi=\sqrt{\frac{1+\alpha}{1-\alpha}}|\tan P|, \\
& \cos \Delta= \pm \beta\left(\sqrt{1-\alpha^{2}}\right)^{-1}(+ \text { if } P>0),
\end{aligned}
$$

where $\theta_{k}$ is the Kerr rotation, $\eta_{k}$ is the Kerr ellipticity, $P$ is the polarizer azimuthal angle in the ellipsometer with respect to the plane of incidence ( $p$ plane), and $\alpha$ and $\beta$ are given by

$$
\begin{aligned}
& \alpha=\frac{\left|R_{p}\right|^{2} \cos ^{2} P-\left|R_{s}\right|^{2} \sin ^{2} P+2\left[\operatorname{Re}\left(\widetilde{R}_{p} \widetilde{K}^{*}\right)-\operatorname{Re}\left(\widetilde{R}_{s} \widetilde{K}^{*}\right)\right] \sin P \cos P}{\left|R_{p}\right|^{2} \cos ^{2} P+\left|\widetilde{R}_{s}\right|^{2} \sin ^{2} P+2\left[\operatorname{Re}\left(\widetilde{R}_{p} \widetilde{K}^{*}\right)+\operatorname{Re}\left(\widetilde{R}_{s} \widetilde{K}^{*}\right)\right] \sin P \cos P}, \\
& \beta=2 \frac{\operatorname{Re}\left(\widetilde{R}_{p} \widetilde{K}^{*}\right) \cos ^{2} P+\operatorname{Re}\left(\widetilde{R}_{s} \widetilde{K}^{*}\right) \sin ^{2} P+\operatorname{Re}\left(\widetilde{R}_{p} \widetilde{R}_{s}^{*}\right) \sin P \cos P}{\left|\widetilde{R}_{p}\right|^{2} \cos ^{2} P+\left|\widetilde{R}_{s}\right|^{2} \sin ^{2} P+2\left[\operatorname{Re}\left(\widetilde{R}_{p} \widetilde{K}^{*}\right)+\operatorname{Re}\left(\widetilde{R}_{s} \widetilde{K}^{*}\right)\right] \sin P \cos P} .
\end{aligned}
$$

Note that the ellipsometric parameters $\psi$ and $\Delta$ are then functions of the magneto-optic quantity $\widetilde{K}$ as well as the optical coefficients $\widetilde{R}_{p}$ and $\widetilde{R}_{s}$. As a result, even in the absence of an applied field the remanent magnetization of the sample will cause a polar Kerr effect, and hence will effect the values of the ellipsometric parameters.

The analysis of these trilayer structures is not possible with only VASE and Kerr data, as the parameter correlation between the $\mathrm{TbFeCo}$ optical constants and the top dielectric layer thickness prevents an unambiguous simultaneous determination of these parameters. ${ }^{5}$ The inclusion of reflectance data in the fit was found to reduce this correlation so that the top dielectric thickness and $\mathrm{TbFeCo}$ constants could be simultaneously determined for each sample.

\section{RESULTS AND CONCLUSIONS}

The optical constants of the TbFeCo layer, shown in Figs. 4 and 5, were found not to vary with layer thickness. The vertical bars in both figures indicate the range of values determined for all samples, i.e., the values of the optical constants of all $\mathrm{TbFeCo}$ layers lie within the vertical bars. There appears to be small variations in the magneto-optical constants $Q 1$ and $Q 2$, as can be seen in Figs. 5 and 6; however, this variation is not systematic in that there is no distinct trend with increasing or decreasing layer thickness, and is considerably larger than the statistical uncertainties of these parameters as found from the regression analysis. As a result, this variation cannot be attributed to varying layer thicknesses or to experimental error.

The major contribution of this work is to provide spectra of both optical and magneto-optical constants for $\mathrm{Tb}$ $\mathrm{FeCo}$ thin films, thus permitting predictive performance modeling calculations to be made for any structure involv- ing similar TbFeCo thin films. Additionally, a powerful analysis technique has been demonstrated whereby readily available reflection mode techniques (ellipsometry, reflectometry, and Kerr spectrometry) can be used to accurately characterize individual magneto-optic films in relatively complicated multilayer structures. We have also shown that the magneto-optic activity of the sample in the remanent state (assuming a square loop material) can be accounted for in the modeling of ellipsometric data in order to provide the most accurate possible optical constants for the layer(s) of interest. Finally, we have demonstrated the lack of systematic thickness dependence of the optical and magneto-optical constants of $\mathrm{TbFeCo}$ thin films over the thickness range $100-800 \AA$.

\section{ACKNOWLEDGMENT}

The research at the University of Nebraska was supported by National Science Foundation Grant No. CMR8918889.

${ }^{1}$ D. S. Bloomberg and G. N. N. Connell, in Magnetic Recording, edited by C. D. Mee and E. D. Daniel (McGraw-Hill, NY, 1988), Vol. II, Chap. 6, p. 305.

${ }^{2}$ S. L. Grove and W. A. Challener, Proc. Jpn. J. Appl. Phys. 28, Suppl. 28-3, 51 (1989).

${ }^{3}$ W. A. McGahan, P. He, L. Y. Chen, and J. A. Woollam (these Proceedings)

${ }^{4}$ W. A. McGahan, L. Y. Chen, J. A. Woollam, F. Sequeda, and H. Do, Appl. Phys. Lett. (to be published).

${ }^{5}$ G. A. N. Connell, Appl. Opt. 22, 3155, 1983.

${ }^{6}$ J. A. Woollam, P. G. Snyder, and M. C. Rost, Mater. Res. Soc. Proc. 93, 203 (1987).

${ }^{7}$ P. G. Snyder, M. C. Rost, G. H. Bu-Abbud, J. A. Woollam, and S. A. Alterovitz, J. Appl. Phys. 60, 3293 (1986).

${ }^{2}$ L. Y. Chen and J. A. Woollam, SPIE 1166, 267 (1989).

${ }^{9}$ W. A. McGahan and J. A. Woollam, Appl. Phys. Comm. 8, 1 (1989). 